Rev. Adm. Saúde (On-line), São Paulo, v. 19, n. 77: e188, out. - dez. 2019, Epub 25 nov. 2019 http://dx.doi.org/10.23973/ras.77.188

ARTIGO ORIGINAL

\title{
Avaliação do funcionamento dos aparelhos de ultrassom terapêutico em serviços públicos e privados da cidade de Manaus
}

Evaluation of the operation of therapeutic ultrasound appliances in public and private services of the city of Manaus

\section{Rogério Olmedija de Araújo1, Raynara Fonsêca dos Santos²}

1. Fisioterapeuta, mestre em ciências da saúde. Professor Adjunto do Departamento de Morfologia da Universidade Federal do Amazonas (UFAM), Manaus AM

2. Fisioterapeuta. Residente do Programa Multiprofissional em Saúde da UFAM, Manaus AM

\section{RESUMO}

Introdução: O ultrassom terapêutico está entre os recursos mais utilizados por fisioterapeutas no tratamento de disfunções do sistema osteomioarticular. 0 insucesso no tratamento com ultrassom é causado quase sempre pela falta de calibração do aparelho que resulta na diminuição ou ausência dos benefícios pretendidos. Objetivo: Verificar o funcionamento dos aparelhos de ultrassom utilizados terapeuticamente em serviços públicos e privados de fisioterapia. Métodos: Coleta de dados por meio do preenchimento da ficha de avaliação após realização do teste de cavitação que tem por objetivo verificar do funcionamento dos aparelhos. Resultados: Foram avaliados 28 aparelhos, tendo obtido negativo no teste de cavitação um total de 5 aparelhos, que estavam em uso contínuo nos estabelecimentos. Conclusão: Assim, recomenda-se a aferição frequente do funcionamento dos aparelhos por meio do teste de cavitação, sendo um recurso de baixo custo que promove maior confiabilidade, resultando em tratamentos mais eficazes.

Palavras-chave: Terapia por Ultrassom; Cavitação; Calibragem; Fisioterapia 


\section{ABSTRACT}

Introduction: Therapeutic ultrasound is among the most used resources by physiotherapists in the treatment of osteomioarticular system disorders. Failure in ultrasound treatment is almost always due to the lack of calibration of the device that results in decreased or no desired benefits. Objective: To verify the operation of ultrasound devices used therapeutically in public and private physiotherapy services. Methods: Data collection by completing the evaluation form after the cavitation test that aims to verify the functioning of the devices. Results: Twenty-eight devices were applied, with negative effects on cavitation testing in a total of 5 devices, which were in continuous use in the applications. Conclusion: Thus, it is recommended to frequently check the functioning of the devices through cavitation testing, being a low-cost feature that promotes greater use, resulting in more difficult use.

Keywords: Ultrasound Therapy; Cavitation; Calibration; Physiotherapy

\section{INTRODUÇÃO}

O ultrassom terapêutico (UST) está entre os recursos mais utilizados por fisioterapeutas no tratamento de disfunções do sistema osteomioarticular ${ }^{1}$. O UST é um recurso capaz de gerar uma energia sonora que é produzida por uma corrente alternada que flui por um cristal piezoelétrico, alojado em um transdutor, gerando um efeito mecânico que, em tecidos biológicos, é capaz de produzir alterações celulares ${ }^{2}$.

Dentre os efeitos fisiológicos causados pelo UST estão a angiogênese, regeneração tissular, reparação dos tecidos moles, aumento na circulação sanguínea, liberação de macrófagos, síntese de proteína, ativação do ciclo de cálcio e, consequentemente, aumento da mobilidade articular e a extensibilidade em tecidos ricos em colágeno, redução de espasmos musculares e alívio de dor. Esses efeitos justificam a sua indicação para diversas disfunções articulares como osteoartrite e bursite, aceleração na consolidação de fraturas, epicondilites, bem como melhora a penetração de medicamentos ${ }^{1}$.

O UST pode ter efeitos térmicos e não térmicos. Os efeitos térmicos são produzidos por ondas contínuas, resultando em elevação da temperatura tecidual. Em contrapartida, os efeitos não térmicos são produzidos por ondas pulsadas, com intervalos na emissão, e resultam do efeito mecânico, causando alterações como micromassagem e cavitação internamente nos tecidos ${ }^{3}$.

A transmissão de energia do ultrassom (US) necessita de um meio condutor que deverá ser interposto entre o transdutor e a pele do paciente. Essa substância deverá ter propriedades ótimas de condução, sendo comumente 
oferecida no mercado em forma de gel, pois possui pelo menos três propriedades físicas similares à água. Já a água em seu estado líquido é tida como a melhor interface de acoplamento, conduzindo $100 \%$ das ondas mecânicas geradas contra $96 \%$ do meio gel ${ }^{4}$.

O transdutor do UST pode ter duas frequências, e isso está diretamente relacionado com a absorção e atenuação do feixe ultrassônico: $3 \mathrm{MHz}$ é a frequência mais superficial, atingindo profundidades de 1 a $2 \mathrm{~cm}$, direcionado até o tecido subcutâneo; $1 \mathrm{MHz}$ atinge tecidos mais profundos, de 3 a $5 \mathrm{~cm}^{3,4}$.

Mesmo com seus efeitos biológicos registrados na literatura como benéficos, pesquisas mostram grande número de insucesso no tratamento com UST, sendo este fator causado quase sempre pela falta de calibração do aparelho que resulta na diminuição ou ausência dos benefícios pretendidos, gerando altos custos e tratamentos prolongados, sem mencionar a perda de tempo para ambos, Fisioterapeuta e paciente, devido ao uso de um UST placebo' ${ }^{1}$.

Placebo é uma intervenção projetada para simular a terapia médica, não acreditada para ter um efeito específico na doença ou na circunstância a que está sendo aplicado 5 . O efeito placebo é obtido quando ocorre um resultado positivo do uso de um placebo e é preciso que se acredite que algo positivo irá acontecer. Além do efeito placebo há o nocebo que consiste no aparecimento de um efeito colateral ou piora do estado inicial do paciente 6 . No caso específico do UST, a falha na emissão de suas ondas mecânicas, pode tornarse um placebo na sua aplicação.

O placebo é utilizado por estudiosos com diversos fins. No entanto, há uma ética que rege seu uso e pressupõe dois conceitos básicos de controle: placebo como objetivo de um engano planejado, de preferência cego, para ambos investigadores e pacientes ou placebo para retardar a utilização de uma droga reconhecidamente eficaz ${ }^{6}$.

Diversos métodos que podem ser utilizados para mensurar a energia do campo acústico, sua intensidade e grandezas derivadas, podendo ser térmicos, mecânicos, ópticos, elétricos e químicos ${ }^{1}$. A literatura ainda descreve como bons os procedimentos de medição por hidrofone. Além destes, outro método eficaz na análise do campo acústico é o teste de cavitação, que indica a emissão ou não da energia ultrassônica e consiste em colocar água sobre o transdutor e, após ligar o aparelho no modo contínuo, deve-se observar a cavitação numa intensidade a partir de $0,1 \mathrm{~W} / \mathrm{cm}^{2}$, e nebulização da água com o aumento da intensidade entre 1,3 a $1,8 \mathrm{~W} / \mathrm{cm}^{2}$, isto é, desde que a frequência de oscilação da cerâmica esteja próxima da normalidade ${ }^{4,7}$.

Calibração é um conjunto de operações que estabelece a relação entre os valores indicados por um instrumento ou sistema de medição e os valores correspondentes das grandezas estabelecidos por padrões. É de suma importância para a qualidade no processo produtivo e deve ser uma atividade normal de produção, pois reduz a variação das especificações técnicas dos produtos e, principalmente, previne defeitos ${ }^{8}$. 
Há uma controvérsia sobre qual o intervalo de tempo ideal para que os UST sejam enviados para calibração. Em vários países há normas que sugerem que a calibração seja feita a cada 3 ou 6 meses, outros sugerem calibração anual, mensal ou mesmo, semanal ${ }^{9}$. No Brasil, no entanto, não se encontra na literatura dados referentes à calibração, exceto em manuais de instrução de uso de alguns aparelhos que sugerem que o usuário faça inspeção e manutenção preventiva a cada 12 meses de utilização do equipamento ${ }^{10}$.

Deste modo, o presente estudo tem o intuito de verificar o funcionamento dos aparelhos de US utilizados terapeuticamente em serviços públicos e privados de fisioterapia, haja visto os grandes benefícios que este recurso pode trazer em relação à cicatrização tecidual, modulação de processo inflamatório, entre outros.

\section{MATERIAL E MÉTODOS}

Esta pesquisa caracteriza-se como um estudo do tipo transversal de cunho quantitativo. Nove instituições públicas que oferecem serviços de fisioterapia vinculadas à Secretaria Municipal de Saúde (SEMSA), e três instituições privadas, totalizando doze estabelecimentos de fisioterapia com a oferta desse recurso. Todas as coletas foram realizadas em locais públicos e privados que oferecem serviços de fisioterapia.

Participaram da coleta de dados clínicas públicas ou privadas que tinham serviço de fisioterapia; que utilizam US; que aceitaram participar da pesquisa e tenham assinado o Termo de Anuência ou serviços que tenham documento da gestão autorizando a realização da pesquisa (no caso dos serviços públicos).

Foram excluídas clínicas públicas ou privadas que não aceitaram participar da pesquisa; que não haviam assinado o Termo de Anuência ou serviços que não possuíam documento da gestão autorizando a realização da pesquisa; que não utilizam US; US que estivessem queimados, impedindo a avaliação; US que, no momento da avaliação, apresentassem sinais de mau contato.

A coleta deu-se por meio do preenchimento da ficha de avaliação após realização do teste de cavitação que tem por objetivo verificar do funcionamento dos UST. O teste é feito colocando-se fita adesiva ao redor do transdutor, deixando um espaço acima deste, de modo que fique no formato de um copo e que a água a ser colocada no transdutor não escoe. A água foi colocada até cobrir a superfície do transdutor. Após o aparelho ser programado em modo contínuo, na frequência de $1 \mathrm{MHz}$, foi ligado e buscou-se observar a nebulização da água com o aumento da intensidade, indicando que o aparelho está funcionando corretamente. No caso de ausência de nebulização da água, concluiu-se que o aparelho não está emitindo as ondas mecânicas e, portanto, não está funcional.

Os dados foram analisados a priori, por meio de estatística descritiva. Todos os dados foram computados e analisados no programa SPSS, versão 16.0, utilizando-se um nível de significância com p menor que 0,05 na comparação 
do teste de cavitação entre aparelhos de UST utilizados na esfera pública e privada, bem como na comparação de entre o tempo de uso destes aparelhos e o teste de cavitação.

Prezando pelas empresas e serviços públicos de saúde, cujos responsáveis autorizaram a pesquisa, todos os dados e informações coletados foram mantidos em sigilo, não sendo expostos, ficando somente sob conhecimento do pesquisador.

\section{RESULTADOS}

A amostra foi composta por 12 clínicas de fisioterapia, sendo no total avaliados 28 aparelhos de ultrassom terapêutico. Deste total, 10 (35,7\%) estavam em uso na esfera privada e $18(64,3 \%)$ na esfera pública, conforme discriminado nas Tabelas 1 e 2.

Tabela 1. Caracterização da amostra da esfera pública.

\begin{tabular}{lcr}
\hline Esfera pública & n & Potência máxima \\
\hline Marca lbramed & 07 & $2 \mathrm{w} / \mathrm{cm}^{2}$ \\
Marca Carci & 05 & $2 \mathrm{w} / \mathrm{cm}^{2}$ \\
Marca HTM & 06 & $2 \mathrm{w} / \mathrm{cm}^{2}$ \\
Total & 18 & - \\
\hline
\end{tabular}

Tabela 2. Caracterização da amostra da esfera privada.

\begin{tabular}{lrr}
\hline Esfera privada & $\mathbf{n}$ & Potência Máxima \\
\hline Marca lbramed & 09 & $2 \mathrm{w} / \mathrm{cm}^{2}$ \\
Marca Quark & 01 & $1,87 \mathrm{w} / \mathrm{cm}^{2}$ \\
Total & $\mathbf{1 0}$ & - \\
\hline
\end{tabular}

Nas Tabelas 3 e 4 estão descritas as quantidades de aparelhos quanto ao tempo de uso e quanto à presença do selo de calibração, respectivamente. Dos 28 aparelhos analisados, $20(71,4 \%)$ tinham tempo de uso maior que 3 anos e 
$8(28,6 \%)$ tempo de uso menor que 3 anos, e 22 (78,6\%) tinham selo de calibração válido e $6(21,4 \%)$ ausente/inválido.

Tabela 3. Tempo de uso dos aparelhos.

\begin{tabular}{lrr}
\hline Tempo de uso & $>3$ anos & $<3$ anos \\
\hline Esfera privada & 02 & 08 \\
Esfera pública & 18 & 0 \\
Total & 20 & 08 \\
\hline
\end{tabular}

Tabela 4. Selo de calibração.

\begin{tabular}{lrrrr}
\hline Selo de Calibração & Válido & Inválido & Ausente & Total \\
\hline Esfera Privada & 5 & 0 & 5 & 10 \\
Esfera Pública & 17 & 0 & 1 & 18 \\
Total & 22 & 0 & 6 & 28 \\
\hline
\end{tabular}

Nas Tabelas 5 e 6 estão descritos os resultados do teste de cavitação correlacionando-os com as esferas administrativas pertencentes e com seu tempo de uso, respectivamente.

Tabela 5. Correlação entre os testes de cavitação (positivo ou negativo) e as esferas administrativas nas quais tais aparelhos se encontravam.

\begin{tabular}{lcr}
\hline Esfera administrativa & $\begin{array}{r}\text { Testes de } \\
\text { cavitação } \\
\text { negativo }\end{array}$ & $\begin{array}{r}\text { Testes de } \\
\text { cavitação } \\
\text { positivo }\end{array}$ \\
\hline Esfera pública & 04 & 14 \\
Esfera privada & 01 & 09 \\
Total & 05 & 23
\end{tabular}

Nota: o teste de qui-quadrado apresentou significância igual a 0,418. 
Tabela 6. Correlação entre o tempo de uso (idade) dos aparelhos de ultrassom e os testes de cavitação (positivo ou negativo).

Tempo de uso (idade) do aparelho

Testes de cavitação negativo

Maior que 3 anos

04

Menor que 3 anos

01

Total

05

Nota: o teste de qui-quadrado apresentou significância igual a 0,640.

\section{DISCUSSÃO}

Os efeitos clínicos do ultrassom são dependentes da dose. Intensidades muito baixas podem não ser suficientes para gerar alterações fisiológicas.

Intensidades mais elevadas atingem tecidos mais profundos, garantindo que a dose programada chegue até o tecido alvo ${ }^{1}$. Os resultados demonstraram que 27 dos aparelhos avaliados possuíam intensidades de até $2 \mathrm{w} / \mathrm{cm}^{2}$, e apenas 1 possuía intensidade de $1,87 \mathrm{w} / \mathrm{cm}^{2}$, sendo ambas consideradas altas intensidades e indicadas para alcançar tecidos profundos.

$\mathrm{Na}$ Tabela 3 os resultados demonstram que 20 aparelhos avaliados estão em uso superior a 3 anos, e apenas 8 aparelhos estão em uso inferior a 3 anos, e que não houve selos inválidos. No entanto, 6 dos 28 aparelhos avaliados não possuíam selo de calibração. Destes, 5 eram da esfera privada, o que pode ser justificado pelo fato de que a maior parte dos aparelhos privados tem menos de 3 anos de uso e ainda não foi demandada manutenção de parte destes aparelhos, daí a ausência do selo em parte significativa deles.

Apesar de não haver consenso sobre a periodicidade da calibração dos aparelhos, é recomendado pelos fabricantes em seus manuais que se siga orientação da ANVISA de manutenção com a periodicidade de 12 meses $^{10}$.

$\mathrm{Na}$ Tabela 5 nota-se uma quantidade significativa de aparelhos do serviço público com falha no teste de cavitação quando comparado ao serviço privado. Tal resultado pode estar relacionado ao tempo de uso dos aparelhos, conforme demonstrado na Tabela 6, onde 4 dos 5 aparelhos cujo teste de cavitação deu negativo têm tempo de uso superior a 3 anos, sendo todos pertencentes ao serviço público, cujo percentual dos aparelhos em uso superior a 3 anos é de $100 \%$. Este achado sugere que o tempo de uso leva ao desgaste e às falhas, sendo essa diferença significativamente maior quando comparado aos aparelhos mais novos.

O presente estudo encontrou limitações, pois não há na literatura estudos semelhantes, e de acordo com os resultados obtidos, eles são aplicáveis 
apenas a essa amostra, não podendo-se inferir que estes serão similares em outras amostras e outros estabelecimentos que utilizem esse recurso. Devido a essa escassez de estudos, recomenda-se que mais pesquisas sejam realizadas na área, a fim de verificar se em demais localidades e outros estabelecimentos os resultados vão de encontro aos aqui encontrados, destacando-se ainda o fato de não haver consenso a respeito da periodização da manutenção dos aparelhos de ultrassom, sendo a manutenção um fator essencial para assegurar o bom funcionamento dos aparelhos, haja visto que foram encontrados no presente estudo aparelhos que não estavam funcionais, porém, estavam sendo utilizados nas clínicas para tratamentos dos pacientes, resultando em terapêutica possivelmente ineficaz.

Assim, o presente estudo demonstrou que clínicas de fisioterapia podem apresentar aparelhos de ultrassom não funcionais em utilização nos seus atendimentos, e neste sentido o teste de cavitação mostrou-se eficiente como teste de verificação de funcionamento. Mesmo que o teste de cavitação forneça apenas um resultado qualitativo, a aferição frequente do funcionamento dos aparelhos por meio dele deve ser adotada, sendo um recurso de baixo custo que promove maior confiabilidade, resultando em tratamentos mais eficazes, haja visto que foram encontrados aparelhos com falha, porém, com selo de calibração válidos.

Vale ressaltar que o teste de cavitação não garante que a dose programada seja a mesma emitida, sendo necessário um procedimento de calibração para obter maior confiabilidade. A calibração garante que os equipamentos não apresentem alterações na intensidade acústica, resultando em tratamentos eficazes.

Considerando a escassez de estudos utilizando-se do teste de cavitação como meio de verificação do funcionamento dos aparelhos de ultrassom terapêutico, além da falta de padronização na periodicidade da calibração dos aparelhos, percebe-se a necessidade de novos estudos que corroborem e demonstrem a necessidade da avaliação periódica dos aparelhos em uso na prática clínica.

\section{REFERÊNCIAS}

1. Ferrari CB. Avaliação do desempenho de equipamentos de ultra-som terapêutico. [Dissertação]. Piracicaba: Universidade Metodista de Piracicaba; 2008.

2. Mendonça AC, Ferreira AS, Barbieri CH, Thomazine JA, Mazzer N. Efeitos do ultra-som pulsado de baixa intensidade sobre a cicatrização por segunda intensão de lesão cutâneas totais em ratos. Acta Ortop Bras. 2006;14(3):152-7.

3. Farcic TS, Lima RM, Machado AF, Baldan CS, Villicev CM, Esteves I et al. Aplicação do ultrassom terapêutico no reparo tecidual do sistema 
musculoesquelético. Arquivos Brasileiros de Ciências da Saúde. 2012 Dez; 37(3):149-53.

4. Agne JE. Eu sei eletroterapia. 4르. ed. Santa Maria: Pallotti; 2009.

5. Dourado E, Fernandes TCA, Meireles JSS, Monteiro IS. Dor e efeito placebo. Rev. Cir. Traum. buco-maxilo-facial. 2004 Set; 4(3):197-203.

6. Soares CP. O efeito placebo. [Monografia]. Brasília: Centro Universitário de Brasília; 2002.

7. Weimann L. Análise da eficácia do ultra-som terapêutico na redução do fibro edema gelóide. [Trabalho de Conclusão de Curso]. Cascavel: Universidade Estadual do Oeste do Paraná; 2004.

8. Inmetro. Vocabulário Internacional de Metrologia: Conceitos fundamentais e gerais e termos associados. $1^{\underline{a}}$ ed. Rio de Janeiro: Inmetro, 2012.

9. Martins FLM, Grosso D, Lima LC, Duarte J. Calibração de ultra-som terapêutico pelo método da balança analítica, In: II Congresso Nacional de Engenharia Mecânica. 2002 agosto 12-16; João Pessoa. João Pessoa: Conem, 2002.

10. Anvisa. Indústria Brasileira de Equipamentos Médicos. Instruções de uso: Sonopulse III. $3^{\underline{a}}$ ed. São Paulo, 2012.

Recebido: 22 de setembro de 2019. Aceito: 22 de novembro de 2019

Correspondência: Rogério Olmedija de Araújo. E-mail:

olmedija_fisio@yahoo.com.br

Conflito de Interesses: os autores declararam não haver conflito de interesses.

(C) This is an Open Access article distributed under the terms of the Creative Commons Attribution License, which permits unrestricted use, distribution, and reproduction in any medium, provided the original work is properly cited 